\title{
Preferential Perpendicular Acceleration of Heavy Ionospheric Ions by Interactions With Electrostatic Hydrogen Cyclotron Waves
}

\author{
NAGENDRA SiNGH, R. W. SCHUNK, AND J. J. SOJKA
}

\author{
Center for Atmospheric and Space Sciences, Utah State University, Logan, Utah 84322
}

\begin{abstract}
The energization of heavy ionospheric ions by electrostatic hydrogen cyclotron (EHC) waves is examined. In particular, the problem of preferential perpendicular acceleration of heavy ions above $5000 \mathrm{~km}$ along auroral field lines is examined critically. Observations have indicated that above this altitude the occurrences of ion beams and EHC waves are highly correlated and that the occurrence rates are high. The EHC waves are found to be coherent. The energization process is examined in the light of interactions of a single coherent wave with the ions. The interactions can be stochastic or nonstochastic, depending on the parameters of the wave and of the background $\mathrm{H}^{+}$ions supporting the wave. With electric field wave amplitudes of a few tens of $\mathrm{mV} / \mathrm{m}$, it is shown that the interaction can give perpendicular energies of the order of $\mathrm{keV}$ for $\mathrm{O}^{+}$ ions. $\mathrm{He}^{+}$ions are shown to gain perpendicular energies smaller than $\mathrm{O}^{+}$ions. It is indicated that upward flowing $\mathrm{H}^{+}$ions cannot gain any appreciable amount of perpendicular energy. For a given electric field wave amplitude $E_{o}$, the extent of energization critically depends on the average perpendicular energy (temperature) $W_{\perp B}$ of the background $\mathrm{H}^{+}$ion plasma. Examples are worked out for values of $W_{\perp B}$ ranging from 1 to $200 \mathrm{eV}$. The resulting energizations and pitch angles for $\mathrm{O}^{+}$and $\mathrm{He}^{+}$ions are compared with observations from satellites. The heating times for the ions are found to be of the order of $2 \mathrm{~s}$ at an altitude of $5000 \mathrm{~km}$.
\end{abstract}

\section{INTRODUCTION}

In recent years there have been numerous observations indicating the presence of energetic ions of ionospheric origin in various parts of the magnetosphere. These energetic ions have been observed at all altitudes. Whalen et al. [1978] observed energetic ions with energy $W \cong 100$ $\mathrm{eV}$ and pitch angle distributions peaking at $90^{\circ}$ at an altitude as low as $500 \mathrm{~km}$. At higher altitudes ( 3500 km), Klumpar [1979] and Ungstrup et al. [1979] reported the occurrence of transversely accelerated ion events, as observed from the ISIS 1 and 2 satellites. Observations at still higher altitudes $\left(\sim 1 R_{E}\right)$ from the S3-3 satellite indicated the presence of ionospheric ions at energies of the order of $\mathrm{keV}$ and with pitch angle distributions either field-aligned or peaking at angles somewhat larger than $90^{\circ}$. This latter pitch angle distribution is indicative of a transverse acceleration mechanism for the ions. Observations from ATS 6 have indicated $\mathrm{H}^{+}$temperatures ranging from a few $\mathrm{eV}$ to several tens of electron volts [Horwitz and Chappell, 1979; Comfort and Horwitz, 1981; Singh et al., 1982a]. Results from the low-energy ion experiments on the ISEE 1 satellite showed ion temperatures of several tens of electron volts in the plasma trough, and the ions were found to have a wide range of pitch angle distributions, from field-aligned and conics to pancake [Baugher et al., 1980; Horwitz et al., 1981]. Energetic ions of ionospheric origin have also been suggested to occur in the distant magnetotail. Frank et al. [1977] reported observations of $\mathrm{H}^{+}$and $\mathrm{O}^{+}$ions drifting away from the earth along geomagnetic field lines with nearly equal speeds of $220 \mathrm{~km} / \mathrm{s}$ at a distance of $35 R_{E}$.

Copyright 1983 by the American Geophysical Union.

Paper number 3A0112.

0148-0227/83/003A-0112\$5.00
Hardy et al. [1977] reported similar observations near the lunar orbit.

The above discussion on the observations of energetic ionospheric ions in various regions of the magnetosphere leads to the basic question regarding the possible mechanisms by which the cold ionospheric ions with energies $E<1 \mathrm{eV}$ are energized. We note that most of the observations discussed earlier pertain to invariant latitudes $\lambda>60^{\circ}$, for which the geomagnetic field lines fall in the auroral or polar cap regions. An outflow of plasma from these regions occurs via the polar wind [Banks and Holzer, 1969]. However, the classical picture of a polar wind flow is a field-aligned plasma flow with drift velocities of typically $10 \mathrm{~km} / \mathrm{s}(\sim 1 \mathrm{eV})$ for $\mathrm{H}^{+}$ions. An in depth understanding of the observations on the energetic ions requires investigations of the modification of the polar wind due to various plasma processes occurring in the polar cap and auroral region.

The recent observations from the S3-3 satellite on particles, parallel and perpendicular dc electric fields, and waves have added greatly to the understanding of the energization of ionospheric ions. One of the most interesting observations has been the finding that ion beams and electrostatic hydrogen cyclotron (EHC) waves are highly correlated and that they show an abrupt increase in their occurrence rate at an altitude of about $5000 \mathrm{~km}$ [Kintner et al., 1979; Gorney et al., 1981]. A statistical survey of upward flowing ion (UFI) events occurring between 6000 and $8000 \mathrm{~km}$ has shown that the average energy of $\mathrm{O}^{+}$ions has a strong correlation with that of the $\mathrm{H}^{+}$ions [Collin et al., 1981]. In a recent study by Lundin et al. [1982] this correlation has been found to exist for UFI events occurring at altitudes extending up to several earth radii. Moreover, a similar correlation between the average energies of $\mathrm{He}^{+}$and $\mathrm{H}^{+}$ions was also found, but $\mathrm{He}^{+}$ions were found to be less energetic than $\mathrm{O}^{+}$ions [Lundin et al., 1982]. 
A statistical survey of the pitch angle distributions of $\mathrm{H}^{+}$and $\mathrm{O}^{+}$ions in UFI events has shown that the beam width of $\mathrm{O}^{+}$ions is larger than that of $\mathrm{H}^{+}$ions [Collin et al., 1981]. Shelley [1979] demonstrated that in UFI events the beam width for $\mathrm{O}^{+}$ions increases with increasing ion energy, while the opposite occurs for $\mathrm{H}^{+}$ions.

The combined observations on the correlation between the energies of $\mathrm{O}^{+}$and $\mathrm{H}^{+}$ions and on the anticorrelation between their pitch angle distributions suggest that in UFI events both ion species undergo a parallel acceleration by field-aligned potential drops. However, the additional energization of the heavy ions is caused by a massdependent preferential acceleration acting primarily in the transverse direction. The observation of a strong correlation between $\mathrm{EHC}$ waves and $\mathrm{H}^{+}$ion beams [Kintner et al., 1979] suggests that the additional transverse acceleration of heavy ions is caused by the interaction of these ions with the EHC waves. Papadopoulos et al. [1980] and Singh et al. [1981, 1982b] have shown that such an interaction can be very effective in the energization of the ions, depending on their mass. The purpose of the present paper is to examine critically the energetics of UFI events in view of the theory of the interaction of a single coherent $\mathrm{EHC}$ wave with $\mathrm{O}^{+}, \mathrm{He}^{+}$, and $\mathrm{H}^{+}$ions.

Assuming that the EHC waves are excited by an interaction between an $\mathrm{H}^{+}$ion beam and a background $\mathrm{H}^{+}$ion plasma, we have calculated the wave potential amplitudes by assuming some reasonable values for the wave electric-field amplitudes, which are available from S3-3 satellite measurements [Kintner et al., 1979; Kintner, 1980; Temerin et al., 1981]. Using these wave potential amplitudes in the theory describing the interaction of coherent waves with charge particles [Fukuyama et al., 1977; Karney, 1978; Smith and Kaufman, 1978], we show that the resulting energies and pitch angles of the ions closely correspond to those observed. An appreciable mass-dependent transverse acceleration of the ions is shown to occur even for very small wave amplitudes, corresponding to wave electric fields $\cong 20 \mathrm{mV} / \mathrm{m}$.

A brief outline of the paper is as follows. The theory of the interaction between charge particles and a coherent wave is reviewed in section 2 . The important features of UFI events that have emerged from observations are pointed out in section 3 . Sections 4,5 , and 6 deal with the energization of $\mathrm{O}^{+}, \mathrm{He}^{+}$, and $\mathrm{H}^{+}$ions, respectively. The energization of ions by EHC waves occurring at the higher harmonics of the hydrogen cyclotron frequency is considered in section 7. A discussion on the transverse energization of ions obtained from theory and its relevance to UFI events is given in section 8 .

\section{Interaction of Charge Particles With A COHERENT WAVE}

A coherent wave propagating at an arbitrary angle with respect to an ambient magnetic field $\mathbf{B}_{o}$ is given by

$$
\phi=\phi_{0} \cos \left(k_{\perp} X+k_{\|} Z-\omega t+\psi\right)
$$

where $\phi$ is the electrostatic potential of the wave, $\phi_{0}$ is the wave amplitude, $\psi$ is the phase constant, $\omega$ is the wave frequency, $k_{\perp}$ and $k_{\|}$are the perpendicular and parallel wave vector components, and $X$ and $Z$ are spatial coordinates, with $Z$ along the magnetic field.

The acceleration of charge particles in a plasma by such a wave was proposed by Stix [1965], and this mechanism was applied to explain the observation of energetic electrons in space plasmas as early as 1965 by Fredericks et $a l$. [1965]. These authors demonstrated that the average gain in perpendicular energy per cyclotron period of a 'favored' charged particle is given by

$$
\Delta W_{\perp} \cong 2 \pi v q \phi_{o} \sin \psi J_{\nu}\left(k_{\perp} r\right) \quad k_{\perp} \gg k_{\|}
$$

where $v$ is an integer satisfying the cyclotron resonance condition

$$
\omega-k_{\|} V_{\|}=\nu \Omega \quad \nu= \pm 1, \pm 2 \text { (integer) }
$$

$J_{v}$ is the Bessel function of order $v, r$ is the Larmor radius of the particle, $\Omega$ is its cyclotron frequency, $q$ is its charge, and $V_{\|}$is its parallel velocity. The 'favored' particle is determined by the phase factor $\psi$.

Since an increment in perpendicular energy implies an increase in the perpendicular velocity $V_{\perp}$ and hence the Larmor radius $r$, (2) shows that the energization of the particle is limited by the zeros of the Bessel function,

$$
J_{v}\left(\zeta=k_{\perp} r\right)=0
$$

For particles with initial energies such that initial values of $\zeta=\zeta_{i} \leqslant \zeta_{1, v}$, where $\zeta_{1, v}$ is the first zero of the Bessel function $J_{v}$, the heating leads to a final value of the Larmor radius $r_{f}$ given by

$$
r_{f} \cong \zeta_{1, v} / k_{\perp}
$$

Later, we show that even this limiting energization corresponding to $r_{f}$ can be significant, especially for heavy ions like $\mathrm{O}^{+}$.

A similar picture of the acceleration of charge particles by coherent waves was presented by Lysak et al. [1980]. They suggested that by adding many more waves to the system, the barriers created by the zeros of the Bessel functions can be avoided. They derived a heating rate given by

$$
\frac{d W_{\perp}}{d t} \cong q \phi_{0} \Omega\left[\frac{2 M \Omega^{2}}{\pi^{2} k_{\perp}^{2} W_{\perp}}\right]^{1 / 4}
$$

where $M$ is the particle's mass. It is worth noting that (6) can be derived from (2) by dividing (2) by the cyclotron period $\tau_{c}=2 \pi / \Omega$ and by replacing $J_{v}$ with its asymptotic expression.

However, we note that it is possible to accelerate charge particles beyond the zeros of the Bessel functions by a single wave of large enough amplitude. The basic idea behind this acceleration process is the overlapping of several resonances. Under such a situation charge particles moving under the influence of a wave are affected by several resonances, and thus, they can move over a larger volume in phase space than when they are affected by only one resonance. An example where the overlapping of resonances can be easily conceptualized is provided by 
the cyclotron resonances in (3), as discussed by Smith and Kaufman [1975, 1978]. The separation between the parallel velocities of these resonances is given by

$$
\Delta V_{\|}^{S}=\Omega / k_{\|}
$$

We now estimate the width of the resonances, which depends upon the wave amplitude. Using the expansion,

$$
\exp \left[k_{\perp} r \sin \Theta\right]=\sum_{n=\infty}^{-\infty} J_{n}\left(k_{\perp} r\right) \exp (\operatorname{in} \Theta)
$$

the wave potential in (1) evaluated at the cyclotron orbit $X=r \sin \Omega t$ can be written as

$$
\phi=\sum_{n=\infty}^{\infty} \phi_{o} J_{n}\left(k_{\perp} r\right) \cos \left(i t k_{\|} V_{\|}+i n \Omega t-i \omega t+i k_{\|} Z_{\mathrm{o}}\right)
$$

where we have substituted $Z=V_{\|} t+Z_{o}, Z_{o}$ being the initial location of the charge particle.

Using the analogy of the motion of a charge particle in a longitudinal wave in the absence of a magnetic field, we see that the resonance half-width is given by

$$
\delta V_{\|} \cong 2\left|q \phi_{\circ} J_{\circ}\left(k_{\perp} r\right) / M\right|^{1 / 2}
$$

When $\phi_{o}$ is large enough so that $\delta V_{\|} \cong \Delta V_{\|} \mathrm{S}$, the resonances overlap. Thus, using (7) and (10), the threshold amplitude for overlapping is found to be [Smith and Kaufman, 1975, 1978]

$$
\alpha \frac{k_{\|}^{2}}{k_{\perp}^{2}}\left|J_{\nu}\left(k_{\perp} r\right)\right| \geqslant \frac{1}{16}
$$

where $\alpha$ contains the amplitude,

$$
\alpha=k_{\perp}^{2} q \phi_{0} / M \Omega^{2}
$$

Thus, starting with a small $V_{\|}$, a particle can be accelerated to a large value of $V_{\|}$, corresponding to the combined width of the overlapping resonances, as long as (11) is satisfied.

However, in the wave frame the total energy of the particle remains constant, giving

$$
\begin{gathered}
\frac{1}{2} M\left[\left(V_{\|}-\omega / k_{\|}\right)^{2}+V_{\perp}{ }^{2}\right] \\
+q \phi_{o} \cos \left(k_{\perp} X+k_{\|} Z-\omega t\right)=\text { const }
\end{gathered}
$$

Under the conditions that the kinetic energy gain $\frac{1}{2}$ $M\left(\Delta V_{\|^{2}}{ }^{2}+\Delta V_{\perp}{ }^{2}\right) \gg>q \phi_{0}$ and the phase velocity $\omega / k_{\|}>>^{2}$ $V_{\|}$, from (13) we see that the change in parallel velocity $\Delta V_{\|}$is related to that in the perpendicular velocity $\Delta V_{\perp}$ according to

$$
\Delta V_{\|}=V_{\|}-V_{\| i}=1 / 2\left(k_{\|} / \omega\right)\left[\left(V_{\perp i}+\Delta V_{\perp}\right)^{2}-V_{\perp i}\right]
$$

where $V_{\| i}$ and $V_{\perp i}$ are the initial velocity components. As the particle is accelerated in the parallel direction, it also gains perpendicular velocity, which leads to an increase in the Larmor radius. Therefore, eventually (11) is no longer satisfied and the acceleration may stop.

Fukuyama et al. [1977] pointed out that when $k_{\perp} \gg k_{\|}$, the threshold criterion in (11) is too restrictive. They derived a threshold criterion given by

$$
\delta \cong v \alpha\left|J_{v}\left(\zeta_{p, v}\right)\right| / \zeta_{p, v} \gtrsim \delta_{t h} \cong 0.15
$$

where $v$ is an integer close to $\omega / \Omega$. The argument $\zeta_{p, v}$ is determined by the maximum of $\left|J_{v}\right|$. Note that $\zeta=k_{\perp} r$. Since the maxima of $\left|J_{v}\right|$ are separated by the zeros of $J_{v}$, we call the range of $\zeta$ between the zeros as cells, numbered by $p$. Depending on the amplitude $\phi_{o}$ (or $\alpha$ ), the threshold criterion (15) can be satisfied over several cells. The upper boundary of the largest cell number satisfying (15) determines the maximum acceleration.

In order to understand the basic difference between the threshold criterions (11) and (15), it is important to understand the modifications in the cyclotron motion due to the wave in (1). Timofeev [1974] demonstrated that under the influence of the wave the rotation frequencies of the particle in the laboratory frame span from the cyclotron frequency $\Omega$ to the wave frequency $\omega$. This nonlinear change in the rotation frequency is crucial to the acceleration mechanism in (15). Such a change in the cyclotron frequency was not considered in the derivation of (11) by Smith and Kaufman [1975, 1978]. When the rotation of the particle has frequencies which can also resonate with the cylindrical harmonics in (9) in addition to $\omega \cong \nu \Omega$ near the zeros of $J_{\nu}\left(k_{\perp} r\right)$, the barriers created by the zeros of the Bessel functions are removed, and particle acceleration in the perpendicular direction is possible as long as (15) is valid.

Karney and Bers [1977] and Karney [1978, 1979] have studied the problem of the acceleration of ions by a coherent lower hybrid wave for which $\omega>>\Omega$ and $k_{\|}<<$ $k_{\perp}$. They showed that it is not important for the ratio $\omega / \Omega$ to be close to an integer for the acceleration to occur. They demonstrated that when $\omega / \Omega$ is far from an integer, the important resonances are of the type $(\omega / \beta) \cong P / S$, where $P$ and $S$ are integers and $\beta$ is the effective rotation frequency due to the nonlinear changes in the cyclotron motion. Several secondary resonances of this type can occur, and when there are enough of them, particles can be accelerated. The threshold criterion for this to happen is similar to (15), except that Karney [1978] estimated $\delta_{t h}$ $\cong 1 / 4$. Our numerical calculations [Singh et al., 1981, $1982 b$ ] are in good agreement with this threshold value.

Karney pointed out that the threshold conditions (11) and (15) are complementary, and for arbitrary values of the ratio $\left(k_{\perp} / k_{\|}\right)$the threshold criterion can be written as

$$
\alpha>\operatorname{Min}\left[\frac{\zeta_{p, v}}{4 v}\left|J_{\nu}\left(\zeta_{p, v}\right)\right|^{-1}, \frac{1}{16} \frac{k_{\perp}}{k_{\|^{2}}^{2}}\left|J_{\nu}\left(\zeta_{p, v}\right)\right|^{-1}\right]
$$

Thus, when $k_{\|} r>\frac{1}{2} \sqrt{v k_{\perp} r}$ (11) is valid, otherwise (15) should be used.

We remark that when particles are influenced by several overlapping resonances, their motions become a random walk in phase space. Therefore, the above acceleration mechanism is called stochastic even though the wave involved in the acceleration is a coherent monochromatic wave. 
Besides the threshold criterion on the wave amplitude given by (15), a charge particle can get accelerated only when its initial velocity $V_{\perp}>\omega / k_{\perp}$, the perpendicular phase velocity of the wave [Karney, 1978]. However, owing to the finite wave amplitude, the limit on $V_{\perp}$ is lowered by the trapping width of the wave. Thus, in order to undergo acceleration, particles must have their initial perpendicular velocity given by [Karney, 1978]

$$
V_{\perp} \gtrsim V_{\perp 0}=\frac{\omega}{k_{\perp}}(1-\sqrt{\alpha} / v)
$$

We note that as the particles are accelerated, their Larmor radius increases, and hence $\alpha$ increases, causing $J_{v}$ to decrease. Thus, the threshold criterion is satisfied up to a limited value $V_{\perp \max }$, which depends on the wave parameters. The region $V_{\perp_{0}}<V_{\perp}<V_{\perp \max }$ will be called the stochastic region in this paper.

The observations from the S3-3 satellite indicate the occurrences of strong coherent electrostatic hydrogen cyclotron (EHC) waves at altitudes above $5000 \mathrm{~km}$ in the auroral region [Kintner et al., 1978, 1979; Temerin et al., 1981]. These waves propagate in a nearly perpendicular direction $\left(k_{\|}<<k_{\perp}\right)$. Thus, the acceleration of ionospheric ions by such a wave will be determined by the threshold criterion (15). Papadopoulos et al. [1980] applied the theory of Karney [1978] to predict a large acceleration of ionospheric ions, such as $\mathrm{O}^{+}$and $\mathrm{He}^{+}$, by EHC waves. For a sufficiently large wave amplitude, $\alpha$ can be large. Thus, by replacing the Bessel function in (15) with its asymptotic expression and by using $\delta_{\text {th }}=1 / 4$, the maximum energy after acceleration, $E_{\max }=1 / 2 M(i) V^{2}{ }_{\max }$, can be expressed as

$$
\begin{gathered}
E_{\max }=3 \cdot 2\left(2 k_{\perp} r_{\mathrm{H}} / \pi\right)^{2 / 3} \phi_{o} 4 / 3 \\
\left(M(i) / m_{\mathrm{H}}\right)^{5 / 3}(e / q)^{2 / 3} k_{\mathrm{B}} T_{\mathrm{H}}
\end{gathered}
$$

where $M_{\mathrm{H}}, T_{\mathrm{H}}$, and $r_{\mathrm{H}}$ are the mass, temperature, and Larmor radius of the hydrogen ions supporting the EHC wave, $M(i)$ and $q$ are the mass and charge of the ion being accelerated, and $k_{\mathrm{B}}$ is the Boltzmann constant. The Larmor radius is $r_{\mathrm{H}}=+\left(k_{\mathrm{B}} T_{\mathrm{H}} / M_{\mathrm{H}}\right)^{1 / 2} / \Omega_{\mathrm{H}}$, where $\Omega_{\mathrm{H}}$ is the gyrofrequency of hydrogen ions. The normalized wave amplitude is represented by $\Phi_{0}\left(\Phi_{0}=e \phi_{0} / k_{\mathrm{B}} T_{\mathrm{H}}\right)$.

It is important to point out that in deriving (18), $v$ is replaced by $M(i) / M_{\mathrm{H}}$, giving the mass dependence in (18). It is worth mentioning that (18) is an excellent approximation for predicting the heating of heavy ionospheric ions, such as $\mathrm{O}^{+}$and $\mathrm{O}^{++}$. On the other hand, it underestimates the heating of light ions when cyclotron resonance effects are present. In the absence of cyclotron resonances, (18) is valid even for light ions [Singh et al., $1981,1982 b]$.

\section{ObSERVATIONS OF UFI EventS AND Stochastic ACCELERATION}

Observations from the S3-3 satellite have shown that the energetic ions with energies of the order of $\mathrm{keV}$ flowing upward along the auroral field lines are an important feature of the auroral plasma above about $5000 \mathrm{~km}$. As far as the composition of these upward flowing ions is concerned, the ions are predominantly $\mathrm{H}^{+}$and $\mathrm{O}^{+}$ However, $\mathrm{He}^{+}$ions are also observed [Lundin et al. 1982]. The pitch angle distributions of the ions ranged from being field-aligned (ion beams) to conics, in which case the flux peaks at an angle to the geomagnetic field. Some important features of the beams and conics are as follows:

1. A statistical survey [Gorney et al., 1981] indicated that the occurrence rate of energetic ion beams increases abruptly at about $4000 \mathrm{~km}$ and then continues to increase above this altitude.

2. A similar observation was made by Kinter et al. [1979] regarding EHC waves, which show a strong correlation with ion beams. It is likely that the ion beams are formed by field-aligned potential drops and the EHC waves are excited by the $\mathrm{H}^{+}$ion beams. Surveys have indicated that the ion beam energy and EHC wave amplitude correlate strongly (P. M. Kintner, private communications, 1982).

3. Gorney et al. [1981] showed that during disturbed times $(K p \gtrsim 3)$ the occurrence rate of ion conics shows a systematic increase with altitude above $4000 \mathrm{~km}$, especially in the dusk sector where the ion beam occurrence rate also peaks. Thus, it appears that during disturbed times the generation of conics is facilitated by the transverse acceleration of the ions by EHC waves generated by $\mathrm{H}^{+}$ion beams.

4. Collin et al. [1981] have carried out a statistical survey correlating the average energies of $\mathrm{O}^{+}$and $\mathrm{H}^{+}$ions and their beam widths for UFI events between altitudes of 6000 and $8000 \mathrm{~km}$. They showed a systematic variation of the average energy of $\mathrm{O}^{+}$ions with that of the $\mathrm{H}^{+}$ions; it was found that on the average $\mathrm{O}^{+}$ions were more energetic than the $\mathrm{H}^{+}$ions and typically $\mathrm{W}\left(\mathrm{O}^{+}\right) \cong 1.7$ $\mathrm{W}\left(\mathrm{H}^{+}\right)$, where $\mathrm{W}$ denotes average ion energy. We note that a similar observation has been made by Lundin et al. [1982] regarding UFI events occurring at altitudes much greater than $8000 \mathrm{~km}$.

5. Collin et al. [1981] have made another interesting observation; the median width of the $\mathrm{H}^{+}$ion pitch angle distribution was found to be smaller than that of the $\mathrm{O}^{+}$ ions. These widths were found to be about $10^{\circ}$ for $\mathrm{H}^{+}$ions and $24^{\circ}$ for $\mathrm{O}^{+}$ions, suggesting that $\mathrm{O}^{+}$ions undergo a preferential perpendicular acceleration in addition to the parallel acceleration by the field-aligned potential drop, which accelerates all equally charged ions by the same amount.

6. Preferential mass dependent perpendicular acceleration of heavy ions is also suggested by the observations from the Prognoz 7 satellite at altitudes beyond $8000 \mathrm{~km}$, as discussed by Lundin et al. [1982]. These authors found that in any UFI event $\mathrm{He}^{+}$ions were more energetic than $\mathrm{H}^{+}$ions, but less energetic than $\mathrm{O}^{+}$ions.

Figure 1 shows schematically the acceleration processes affecting the light and heavy ions. It is suggested that all types of ions undergo a parallel acceleration, while only the heavy ions undergo an appreciable perpendicular acceleration. The above points from (1) to $(6)$ relating to the observations strongly suggest that $\mathrm{EHC}$ waves play a strong role in the preferential perpendicular acceleration of the heavy ionospheric ions observed at altitudes greater than about $5000 \mathrm{~km}$. Theoretical works have shown the possibility of such an acceleration 


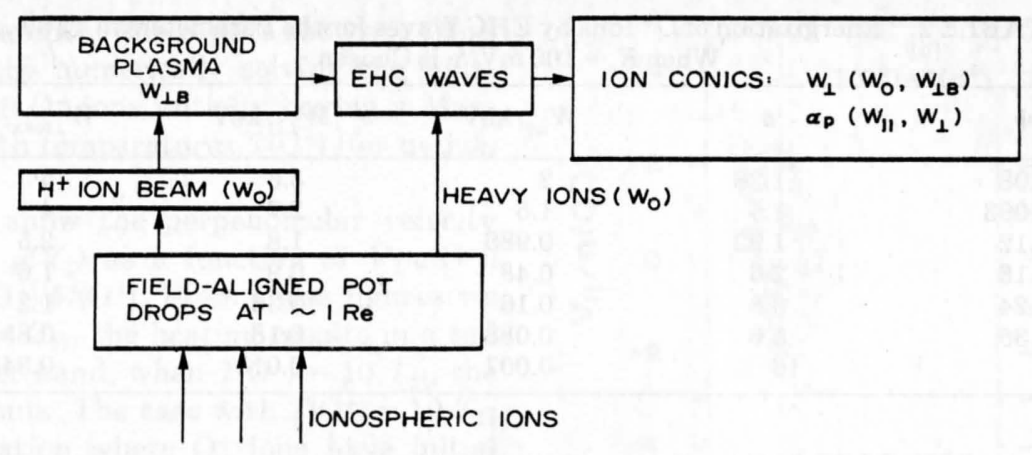

Fig. 1. A schematic diagram showing the parallel acceleration of ionospheric ions followed by a transverse acceleration of heavy ions by EHC waves excited by the $\mathrm{H}^{+}$ion beam.

[Papadopoulos et al., 1980; Singh et al., 1981, 1982b]. We now attempt to examine whether or not the theory of the acceleration detailed in section 2 can explain some of the observed features of the preferential perpendicular acceleration of heavy ions, such as $\mathrm{O}^{+}$and $\mathrm{He}^{+}$.

Our discussion in section 2 showed that in order to estimate the degree of energization we must know the wave parameters and the Larmor radius of the $\mathrm{H}^{+}$ion supporting the wave. The important wave parameters are the wave number $k$ and the amplitude $\phi_{0}$. However, only electric field fluctuations were measured on the S3-3 satellite, which indicated that the electric field amplitudes $E_{0}$ for EHC waves can be larger than 50 $\mathrm{mV} / \mathrm{m}$. In order to estimate $\phi_{0}$ from $E_{0}$, the wave number is required. However, there are no direct measurements of the wavelengths $\lambda=2 \pi / k$. Temerin et al. [1981] estimated that EHC waves with wavelengths as large as $5 \mathrm{~km}$ are possible. Because of the lack of sufficient information on a particular UFI event, we will present some examples of the energization of $\mathrm{O}^{+}$and $\mathrm{He}^{+}$ions by using parameters suitable to the auroral plasma, as indicated by several observations.

Owing to the lack of observations on $\lambda$, the best way to estimate it is on theoretical grounds. Theoretical work on the excitation of ion cyclotron waves by ion beams indicates that such waves are excited by an ion-ion instability [Perkins, 1976], in which the cyclotron mode of the background ions interacts with the resonant-Landau mode of the ion beam to cause growing waves. The parallel wave number and wavelength are given by

$$
k_{\|}=\Omega_{\mathrm{H}} / V_{o} \quad \lambda_{\|}=10^{4} \frac{\sqrt{W_{o} m}}{f_{\mathrm{H}}}
$$

where $V_{0}$ is the beam velocity, $W_{o}$ is the beam energy, and
$\Omega_{\mathrm{H}}\left(=2 \pi f_{\mathrm{H}}\right)$ is the cyclotron frequency of $\mathrm{H}^{+}$ions. The perpendicular wave number and wavelength are

$$
k_{\perp} r_{\mathrm{B}} \cong 1 \quad \lambda_{\perp}=10^{4} \frac{\sqrt{W_{\perp \mathrm{B}}} m}{f_{\mathrm{H}}}
$$

where $r_{\mathrm{B}}$ is the Larmor radius of the background $\mathrm{H}^{+}$ions that have an average perpendicular energy $W_{\perp B}$. An important parameter, which can play an important role in the acceleration of $\mathrm{He}^{+}$ions, is the ratio of perpendicular to parallel wave numbers [Singh et al., 1982b],

$$
k_{\perp} / k_{\|}=\lambda_{\|} / \lambda_{\perp}=\left(W_{o} / W_{\perp \mathrm{B}}\right)^{1 / 2}
$$

Let us assume, for example, that $W_{o}=1 \mathrm{keV}$. At an altitude of about $5000 \mathrm{~km}, f_{\mathrm{H}}=140 H_{z}$. Table 1 shows $\lambda_{\perp}$ and the ratio $k_{\perp} / k_{\|}$when the background $\mathrm{H}^{+}$energy $W_{\perp B}$ is varied over a plausible range from $1 \mathrm{eV}$ to $200 \mathrm{eV}$. In the same table we have given the wave potential amplitude $\Phi_{o}$ for three values of the perpendicular electric field amplitude $E_{0}=20,50$, and $100 \mathrm{mV} / \mathrm{m}$. Note that for these electric field amplitudes the normalized wave potential amplitude $e \phi_{0} / W_{\perp B} \lesssim 1$. The values of the ratio $k_{\perp} / k_{\|}$ indicate the strong effect of cyclotron resonances on the acceleration of relatively light ions such as $\mathrm{He}^{+}[$Singh et al., 1982b].

In the next two sections we present results on the expected energization of $\mathrm{O}^{+}$and $\mathrm{He}^{+}$ions using the theoretical formulas of section 2 and the wave parameters of Table 1.

\section{ENERgization $\mathrm{OF} \mathrm{O}^{+}$IONS}

The parameters which determine the energy gain of the

\begin{tabular}{|c|c|c|c|c|c|}
\hline \multirow[t]{2}{*}{$W_{\perp B}, \mathrm{eV}$} & \multirow[t]{2}{*}{$\lambda_{\perp}, \mathrm{m}$} & \multirow[t]{2}{*}{$\mathbf{k}_{\perp} / \mathbf{k}_{\|}$} & \multicolumn{3}{|c|}{$\Phi_{o}, \mathrm{~V}$} \\
\hline & & & $20 \mathrm{mV} / \mathrm{m}$ & $50 \mathrm{mV} / \mathrm{m}$ & $100 \mathrm{mV} / \mathrm{m}$ \\
\hline 200 & 1000 & 2.2 & 3.2 & 8 & 16 \\
\hline 150 & 875 & 2.6 & 2.8 & 7 & 14 \\
\hline 100 & 715 & 3 & 2.3 & 5.75 & 11.5 \\
\hline 50 & 500 & 4.5 & 1.6 & 4 & 8 \\
\hline 20 & 300 & 7 & 0.95 & 2.4 & 4.8 \\
\hline 10 & 220 & 10 & 0.7 & 1.75 & 3.5 \\
\hline 1 & 70 & 32 & 0.22 & 0.5 & 1 \\
\hline
\end{tabular}
$\mathrm{O}^{+}$ions are given by

TABLE 1. Wave Parameters for Several Values of the Background ion Energy 
TABLE 2. Energization of $\mathrm{O}^{+}$Ions by EHC Waves for the Parameters in Table 1 When $E_{o}=100 \mathrm{mV} / \mathrm{m}$ is Chosen

\begin{tabular}{|c|c|c|c|c|c|c|}
\hline$W_{\perp B}, \mathrm{eV}$ & $\Phi_{0}$ & $\alpha$ & $W_{\perp o}, \mathrm{keV}$ & $W_{\perp 1}, \mathrm{keV}$ & $W_{\perp \max }, \mathrm{keV}$ & $\alpha_{p} \mathrm{o}\left(W_{\mathrm{o}}=1 \mathrm{keV}\right)$ \\
\hline 200 & 0.08 & 1.28 & 2 & 3.6 & 5 & 114 \\
\hline 150 & 0.093 & 1.5 & 1.5 & 2.7 & 4 & 117 \\
\hline 100 & 0.12 & 1.92 & 0.988 & 1.8 & 2.5 & 122 \\
\hline 50 & 0.16 & 2.6 & 0.48 & 0.9 & 1.6 & 128 \\
\hline 20 & 0.24 & 3.8 & 0.16 & 0.36 & 1.2 & 132 \\
\hline 10 & 0.35 & 5.6 & 0.088 & 0.18 & 0.85 & 137 \\
\hline 1 & 1 & 16 & 0.007 & 0.018 & 0.345 & 150 \\
\hline
\end{tabular}

TABLE 3. Same as Table 2, but with $E_{\mathrm{o}}=50 \mathrm{mV} / \mathrm{m}$

\begin{tabular}{|c|c|c|c|c|}
\hline$W_{\perp B}, \mathrm{eV}$ & $\Phi_{0}$ & $\alpha$ & $W_{\perp \max }, \mathrm{keV}$ & $\alpha_{p}\left(W_{o}=1 \mathrm{keV}\right)$ \\
\hline & & Nonstochastic & & \\
\hline 200 & 0.04 & 0.64 & $2.2-3.6$ & 118 \\
\hline 150 & 0.045 & 0.75 & $1.7-2.7$ & 121 \\
\hline 100 & 0.06 & $\begin{array}{c}0.96 \\
\text { Stochastic }\end{array}$ & $1.1-1.8$ & 121 \\
\hline 50 & 0.08 & 1.3 & 1.32 & 131 \\
\hline 20 & 0.12 & 1.9 & 0.64 & 141 \\
\hline 10 & 0.17 & 2.8 & 0.42 & 147 \\
\hline 1 & 0.5 & 8 & 0.154 & 160 \\
\hline
\end{tabular}

TABLE 4. Same as Tables 2 and 3 but with $E_{o}=20 \mathrm{mV} / \mathrm{m}$

\begin{tabular}{rlccc}
\hline$W_{\perp B}$ & $\Phi_{o}$ & $\alpha$ & $W_{\perp \max }, \mathrm{keV}$ & $\alpha_{p}\left(W_{o}=1 \mathrm{keV}\right)$ \\
\hline & & Nonstochastic & \\
100 & 0.016 & 0.26 & $2.2-3.6$ & 118 \\
100 & 0.019 & 0.3 & $1.7-2.7$ & $1.1-1.8$ \\
50 & 0.023 & 0.36 & $0.5-0.91$ & 127 \\
20 & 0.032 & 0.51 & $0.22-0.36$ & 147 \\
10 & 0.0475 & Stochastic & 0.265 & 153 \\
1 & 0.07 & 1.12 & 0.05 & 167 \\
\hline
\end{tabular}

$$
\begin{array}{cc}
M\left(\mathrm{O}^{+}\right) / M_{\mathrm{H}}=16 & \alpha=\mathrm{K}_{\perp} 2 \Phi_{o} M\left(\mathrm{O}^{+}\right) / M_{\mathrm{H}} \\
\nu=\omega / \Omega\left(\mathrm{O}^{+}\right)=19 & \delta=v \alpha\left|J_{v}\left(\zeta_{p, v}\right)\right| / \zeta_{p, v} \gtrsim 1 / 4
\end{array}
$$

where $K_{\perp}=k_{\perp} r_{\mathrm{H}}$ and $\Phi_{0}=\phi_{0} / W_{\perp B}$.

As discussed earlier in section (2), when the threshold condition $\delta>1 / 4$ is satisfied, the ion motion becomes stochastic, and depending on $\Phi_{o}$, appreciable acceleration is possible. The minimum value of $\Phi_{0}$ required to cause stochasticity is determined by the requirement that $\delta>$ $1 / 4$ in at least the first cell of $J_{\nu}(\zeta)$. This amplitude is given by

$$
\Phi_{\min }=\phi_{0} / W_{\perp B}=0.07
$$

For wave amplitudes smaller than the above value, the heating may still occur, but it will be limited to the first zero of $J_{v}(\zeta)$ according to (5). In the following subsections we discuss various aspects of the heating of $\mathrm{O}^{+}$ions.

\subsection{Energetics}

Using (22) and the wave parameters in Table 1 we have determined the maximum possible energy gain $W_{\perp \max }$ for electric field wave amplitudes $E_{o}=100,50$, and $20 \mathrm{mV} / \mathrm{m}$. We note that these values of $E_{0}$ are within the range of observed magnitudes [Kintner et al., 1979; Temerin et al., 1981]. The results of our calculations are given in Tables 2,3 , and 4 . The various entries in the tables are as follows: $W_{\perp B}$ is the average perpendicular energy of the background $\mathrm{H}^{+}$ions, $\Phi_{\circ}$ is the normalized wave potential amplitude, $\alpha$ is defined in (22), $W_{\perp_{0}}$ is the minimum initial ion energy required for stochastic acceleration which is given by (17), $W_{\perp 1}$ is the energy corresponding to the first zero of $J_{v}(\xi), W_{\perp \max }$ is the maximum possible energy gain by the ions, and $\alpha_{\mathrm{p}}$ are the pitch angles for ions having their perpendicular energy equal to $W_{\perp \max }$ and a parallel energy of $1 \mathrm{keV}$.

\subsection{Requirement on the Initial Perpendicular Energy $W_{\perp \circ}$}

The values of $W_{\perp o}$ appearing on Table 2 show that $0^{+}$ ions can undergo the acceleration process only when their initial temperature is about 10 times the ambient $\mathrm{H}^{+}$ temperature. Our numerical calculations have shown that this condition is too restrictive. We have found that an appreciable number of $\mathrm{O}^{+}$ions undergo acceleration even when $T\left(\mathrm{O}^{+}\right)=T_{\mathrm{H}}\left[\right.$ Singh et al., 1981], where $T\left(\mathrm{O}^{+}\right)$and $T_{\mathrm{H}}$ are the initial $\mathrm{O}^{+}$and $\mathrm{H}^{+}$temperatures. For example, the acceleration of ions for a wave amplitude $\phi_{0}=0.4, K_{\perp}=$ 1.7 and for initial $\mathrm{O}^{+}$temperatures $T\left(\mathrm{O}^{+}\right)=T_{\mathrm{H}}$ and $10 T_{\mathrm{H}}$ 
is shown in Figures $2 a$ and $2 b$. The results shown in these figures were obtained by numerically solving the equations of motion of $2000 \mathrm{O}^{+}$ions initially having a Maxwellian distribution with temperatures $\mathrm{T}\left(\mathrm{O}^{+}\right)$(for details see Singh et al. [1981]).

Figures $2 a$ and $2 b$ show the perpendicular velocity distribution functions $g\left(V_{\perp}\right)$ as a function of $V_{\perp} / a\left(\mathrm{O}^{+}\right)$, where $a^{2}\left(\mathrm{O}^{+}\right)=2 k_{B} T\left(\mathrm{O}^{+}\right) / M\left(\mathrm{O}^{+}\right)$. From these figures we note that when $T\left(\mathrm{O}^{+}\right)=T_{\mathrm{H}}$, the heating results in a tail formation. On the other hand, when $T\left(\mathrm{O}^{+}\right)=10 T_{\mathrm{H}}$, the heating occurs in the bulk. The case with $T\left(\mathrm{O}^{+}\right)=10 T_{\mathrm{H}}$ corresponds to a situation where $\mathrm{O}^{+}$ions have initial perpendicular energies of the order of $W_{\perp_{0}}$ given in Table 2.

Comparing the distribution functions in Figure $2 a$ at $t=0$ and $t=80 \tau_{c}\left(\mathrm{O}^{+}\right)$, where $\tau_{c}\left(\mathrm{O}^{+}\right)$is the $\mathrm{O}^{+}$ion cyclotron period, we note that ions with initial perpendicular velocities as small as the ion thermal velocity have been accelerated. This is shown more clearly in Figure $3 a$ by plotting the contours of the distribution function of the initial ion-source population which eventually underwent acceleration. Figure $3 b$ shows a similar plot for the case when $T\left(\mathrm{O}^{+}\right)=10 T_{\mathrm{H}}$. Note that the vertical lines marked with the values of $V_{\perp \circ}$ are obtained from (17), which determined $W_{\perp \circ}$ given in Table 2. The interesting feature of Figure $3 a$ is that the ions with velocities smaller than that determined from (17) can be energized by stochastic acceleration. From Figures $3 a$ and $3 b$ we infer that the requirement for $\mathrm{O}^{+}$ions to undergo acceleration is that their initial energy $W_{\perp 0} \cong W_{\perp B}$.

Assuming that the initial perpendicular energy of $\mathrm{O}^{+}$ ions is nearly the same as that of the ambient $\mathrm{H}^{+}$ions, Table 2 shows that energization leads to a considerable enrgy gain by $\mathrm{O}^{+}$ions, ranging from a factor of 25 at $W_{\perp B}$ $=200 \mathrm{eV}$ to a factor of 345 at $W_{\perp B}=1 \mathrm{eV}$.

Table 3 shows the information on energization of $\mathrm{O}^{+}$

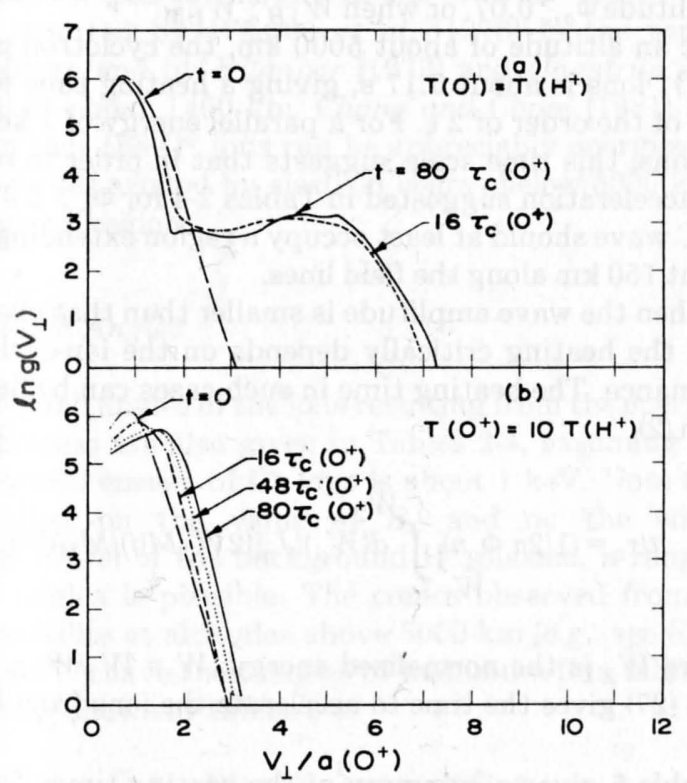

Fig. 2. Stochastic acceleration of $\mathrm{O}^{+}$ions is shown for two values of the initial temperature of $\mathrm{O}^{+}$ions: $(a) T\left(\mathrm{O}^{+}\right)=T_{\mathrm{H}}$, and (b) $T\left(\mathrm{O}^{+}\right)=10 T_{\mathrm{H}}$. The temporal evolution of the acceleration is shown; $\tau_{c}$ is the cyclotron period for $\mathrm{O}^{+}$ions and $g\left(V_{\perp}\right)$ is the perpendicular velocity distribution function.

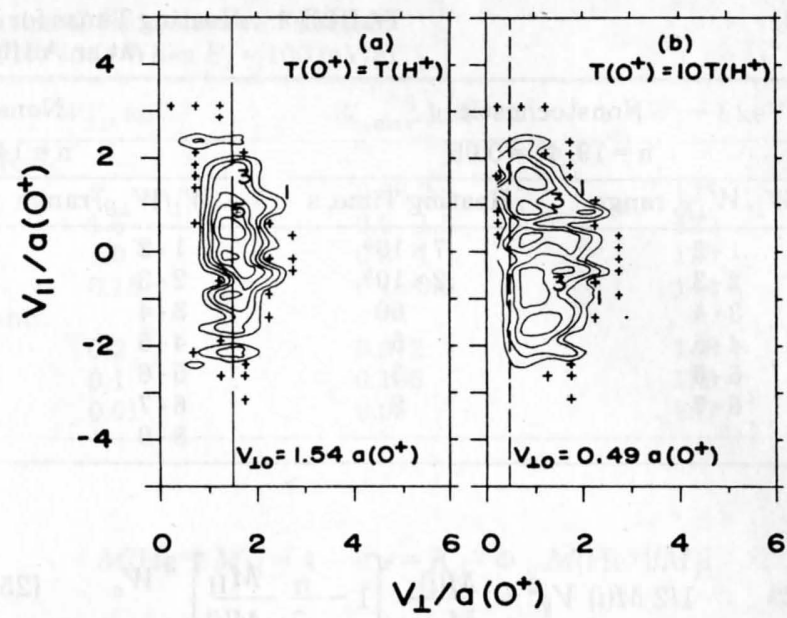

Fig. 3. The initial source population that eventually gets accelerated. The acceleration criterion is chosen so that any particle with $V_{\perp}>2 a(i)$ is assumed to have undergone acceleration. Note that the initial Maxwellian has about $4 \%$ of such particles. Panel (a) is for $T\left(\mathrm{O}^{+}\right) / T_{\mathrm{H}}=1$; note that there are no accelerated particles for $V_{\perp}<0.75 a\left(\mathrm{O}^{+}\right)$, indicating that almost no particles with perpendicular velocity less than $0.75 a\left(\mathrm{O}^{+}\right)$were accelerated. The theoretical limit is $V_{\perp O}=1.54 a\left(\mathrm{O}^{+}\right)$. Panel $(b)$ is for $T\left(\mathrm{O}^{+}\right) / T_{\mathrm{H}}=$ $10, V_{\perp O}=0.49 a\left(\mathrm{O}^{+}\right)$.

ions when $E_{o}=50 \mathrm{mV} / \mathrm{m}$. The values of the normalized wave amplitude indicate that in the present situation the amplitude criterion (23) is satisfied only when $W_{\perp B} \lesssim 50$ $\mathrm{eV}$, implying that stochasticity occurs when the background ions are relatively colder than that for the case of $E_{o}=100 \mathrm{mV} / \mathrm{m}$. Similarly, we note from Table 4 that when the wave amplitude $E_{o}$ is further reduced the stochasticity condition is satisfied only when the background $\mathrm{H}^{+}$ion temperature is also further reduced to $W_{\perp B} \lesssim 10 \mathrm{eV}$. Thus, depending on the electric field wave amplitude there is a maximum value of average perpendicular energy of the background $\mathrm{H}^{+}$ions below which the acceleration process becomes stochastic. We call this maximum $W_{\mathrm{BM}}$.

From Tables 3 and 4 , it is easy to verify that when $W_{\perp B}$ $\ll W_{\mathrm{BM}}$, the acceleration leads to $W_{\perp \max } \sim \Phi_{0} 4 / 3$, as predicted from the theory (see (18)). When $W_{\perp B} \cong W_{\mathrm{BM}}$ the maximum heating is calculated directly from the threshold criterion (22); it is given by the zeros of the Bessel function beyond the first zero.

When $W_{\perp B}>W_{\mathrm{BM}}$, implying that the threshold criterion is not satisfied even in the first cell of $J_{v}(\xi)$, it is still possible to accelerate some ions that eventually will have their Larmor radius corresponding to the first zero of a Bessel function $J_{n}(\xi)$, where $n$ is an integer giving the cyclotron resonance between the wave and the ions:

$$
V_{\|}=\frac{\omega-n \Omega(i)}{k_{\|}}
$$

We emphasize that when the stochasticity condition $\delta>$ $1 / 4$ is satisfied, such resonances are not essential for the acceleration to occur.

We now consider the situation of a UFI event in which even the heavy ions have their parallel energy as $W_{o}$. Using (19) for $k_{\|},(24)$ can be written in terms of the parallel energy of the heavy ions. 
TABLE 5. Heating Times for Nonstochastic and Stochastic Cases at an Altitude of $5000 \mathrm{~km}$

\begin{tabular}{|c|c|c|c|c|c|}
\hline \multicolumn{2}{|c|}{$\begin{array}{c}\text { Nonstochastic } \\
\mathrm{n}=19, \Phi_{\mathrm{o}}=0.05\end{array}$} & \multicolumn{2}{|c|}{$\begin{array}{c}\text { Nonstochastic } \\
\mathrm{n}=14, \Phi_{\mathrm{o}}=0.05\end{array}$} & \multicolumn{2}{|c|}{$\begin{array}{c}\text { Stochastic } \\
\Phi_{0}=0.4\end{array}$} \\
\hline$W_{\perp} / W_{\perp B}$, range & Heating Time, $s$ & $W_{\perp} / W_{\perp B}$, range & Heating Time, $\mathrm{s}$ & $W_{\perp}$ & Heating Time, $\mathrm{s}$ \\
\hline $\begin{array}{l}1-2 \\
2-3 \\
3-4 \\
4-5 \\
5-6 \\
6-7\end{array}$ & $\begin{array}{c}7 \times 10^{5} \\
2 \times 10^{3} \\
60 \\
5 \\
3 \\
2\end{array}$ & $\begin{array}{l}1-2 \\
2-3 \\
3-4 \\
4-5 \\
5-6 \\
6-7 \\
8-9\end{array}$ & $\begin{array}{c}180 \\
8 \\
1.5 \\
0.5 \\
0.2 \\
0.1 \\
0.4\end{array}$ & $W_{\perp B}-W_{\perp \max }$ & $\sim 2$ \\
\hline
\end{tabular}

$$
1 / 2 M(i) V_{\|}^{2}=\frac{M(i)}{M_{\mathrm{H}}}\left[1-\frac{n}{\hat{\omega}} \frac{M_{\mathrm{H}}}{M(i)}\right]^{2} W_{o}
$$

where we recall that $M(i)$ is the mass of a heavy ion and $\hat{\omega}$ $=\omega / \Omega_{\mathrm{H}}$. Using the condition that $W_{\mathrm{o}} \cong 1 / 2 M(i) V_{\|}^{2}$, we find $n$ an integer closest to $12 \hat{\omega}$ for $\mathrm{O}^{+}$and $2 \hat{\omega}$ for $\mathrm{He}^{+}$.

If we assume $\hat{\omega} \cong 1.2$, then $n=14$ for $\mathrm{O}^{+}$ions. However, we note that there may be several cyclotron resonances affecting the heavy ions, giving a range of values of $n$ from 14 to 19 if their parallel velocity distribution function is fairly broad. In Tables 3 and 4 the range of values of $W_{\perp \max }$ for $W_{\perp B}>W_{\mathrm{BM}}$ corresponds to values of $n$ from 14 to 19 . The lowest energy is obtained from the first zero of $J_{14}(\xi)$, while the maximum energy corresponds to the first zero of $J_{19}(\xi)$.

The important point to be noted from Tables 3 and 4 is that even if the stochasticity condition is not satisfied $\left(W_{\perp B}>W_{\mathrm{BM}}\right)$, it is possible to accelerate $\mathrm{O}^{+}$ions to perpendicular energies ranging from about 10 to 18 times $W_{\perp B}$. This energization mechanism is the same as that suggested by Fredericks et al. [1965] and more recently by Lysak et al. [1980] with one coherent wave. An interesting consequence of this nonstochastic acceleration is the generation of $\mathrm{O}^{+}$ions with perpendicular velocities given by

$$
V_{\perp}\left(\mathrm{O}^{+}\right) \cong(1 \text { to } 1.5) V_{\perp \mathrm{B}}\left(\mathrm{H}^{+}\right)
$$

where $V_{\perp \mathrm{B}}$ is the velocity of $\mathrm{H}^{+}$ions with an energy $W_{\perp B}$. Thus, if the background $\mathrm{H}^{+}$plasma is very hot so that $W_{\perp B}>W_{\mathrm{BM}}$, it is possible to generate $\mathrm{O}^{+}$and $\mathrm{H}^{+}$ions with nearly equal perpendicular velocities. If such heavy and light ions flow out of the topside ionosphere to large distances along the geomagnetic field lines, they may emerge as ions having nearly equal parallel velocities. Such a condition has been observed in the distant magnetotail by Frank et al. [1977] and Hardy et al. [1977].

To summarize our discussion so far, we emphasize that if the average perpendicular energy (or equivalently the perpendicular temperature) of the background $\mathrm{H}^{+}$ions $W_{\perp B} \geqq 20 \mathrm{eV}$, it is possible to heat $\mathrm{O}^{+}$ions to perpendicular energies of the order of $\mathrm{keV}$ with electric field wave amplitudes in the range observed by the S3-3 satellite at about $5000 \mathrm{~km}$. In the next section, we examine the time taken to realize the extent of the heating shown in Tables 2-4.

\subsection{Temporal Evolution of the Heating}

A very important aspect of the energization process is the time constant; the question is, how long does it take to energize the ions to the fullest extent? When the normalized wave amplitudes are sufficiently large to cause stochastic heating, the acceleration proceeds quite rapidly. Karney [1978] demonstrated that when $v \cong 30$ and $\alpha$ $\cong 20$, about $75 \%$ of the maximum heating occurs in about 10 ion cyclotron periods. This phase of rapid heating is followed by a slow heating rate. We have found a similar temporal behavior of the heating of $\mathrm{O}^{+}$ions by an EHC wave with wave parameters such that $\alpha \cong 18$ and $\nu=19$. Figure 2 shows the temporal evolution of the perpendicular velocity distribution function $g\left(V_{\perp}\right)$. Note the rapid heating from $t=0$ to $16 \tau_{\mathrm{c}}\left(\mathrm{O}^{+}\right)$and then the modifications in $g\left(V_{\perp}\right)$ with increasing time are very slow. Thus, when the wave amplitude is sufficiently high, implying that the threshold criterion $\delta>1 / 4$ is satisfied in at least the first cell of the Bessel function $J_{v}(\xi)$, most of the heating occurs in about the first ten ion-cyclotron periods. From Tables 2-4 we have already noted that this is possible for $\mathrm{O}^{+}$ions when the normalized wave amplitude $\Phi_{0} \gtrsim 0.07$, or when $W_{\perp B} \lesssim W_{\mathrm{BM}}$.

At an altitude of about $5000 \mathrm{~km}$, the cyclotron period for $\mathrm{O}^{+}$ions is about $0.17 \mathrm{~s}$, giving a heating time for $\mathrm{O}^{+}$ ions of the order of $2 \mathrm{~s}$. For a parallel energy of $1 \mathrm{keV}$ for $\mathrm{O}^{+}$ions, this time scale suggests that in order to realize the acceleration suggested in Tables 2-4 for $\Phi_{0}>0.07$, the $\mathrm{EHC}$ wave should at least occupy a region extending over about $150 \mathrm{~km}$ along the field lines.

When the wave amplitude is smaller than that given by (23), the heating critically depends on the ion-cyclotron resonance. The heating time in such cases can be derived from (2),

$$
t / \tau_{c}=\underset{\substack{\left.\Phi_{o} n\right) \\ \hat{W}_{1}}}{\left(1 / 2 \pi \hat{W}_{2}\right.} d\left(\hat{W}_{\perp}\right) / J_{n}\left[\left(2 W_{\perp} M(i) / M_{\mathrm{H}}\right)^{1 / 2}\right]
$$

where $\hat{W}_{\perp}$ is the normalized energy, $W=W_{\perp} / W_{\perp B}$. Note that (27) gives the time to accelerate the ions from $\hat{W}_{1}$ to $\hat{W}_{2}$.

Table 5 gives a summary of the heating times for two wave amplitudes, $\Phi_{o}=0.05$ and 0.4 ; in the former case the stochasticity condition is not satisfied, while in the latter case it is. When $\Phi_{o}=0.05$, we have evaluated the integral 
TABLE 6. Perpendicular Acceleration of $\mathrm{He}^{+}$Ions as a Function of Perpendicular Average Energy of $\mathrm{H}^{+}$Ions When $E_{0}=100 \mathrm{mV} / \mathrm{m}$

\begin{tabular}{|c|c|c|c|c|c|}
\hline$W_{\perp B}, \mathrm{eV}$ & $\Phi_{0}$ & $\alpha$ & $W_{\perp 1}, \mathbf{k e V}$ & $W_{\perp \max }, \mathbf{k e V}$ & $\alpha_{p}^{\circ}\left(W_{o}=1 \mathrm{keV}\right)$ \\
\hline \multicolumn{6}{|c|}{ Nonstochastic } \\
\hline 200 & 0.08 & 0.32 & 2 & $0.68-2$ & 125 \\
\hline 150 & 0.093 & 0.37 & 1.5 & $0.5-1.5$ & 129 \\
\hline 100 & 0.12 & 0.48 & 1.0 & $0.3-1$ & 135 \\
\hline \multicolumn{6}{|c|}{ Stochastic } \\
\hline 20 & 0.24 & 0.94 & 0.2 & 0.372 & 148 \\
\hline 10 & 0.35 & 1.4 & 0.1 & 0.186 & 156 \\
\hline 1 & 1 & 4 & 0.01 & 0.05 & 167 \\
\hline
\end{tabular}

in (27) numerically, and Table 5 shows results for $n=19$ and 14 corresponding to the energy ranges of $W_{\perp \max }$ given in Tables 3 and 4 for $W_{\perp B}>W_{\mathrm{BM}}$. Note the disparity in the heating times for the stochastic and nonstochastic situations. It was shown earlier that it takes about $2 \mathrm{~s}$ to realize most of the acceleration under the stochastic situation. However, Table 5 shows that the corresponding time under the nonstochastic situation is about $200 \mathrm{~s}$ for $n=14$ and about 20 hours for $n=19$. The disparity in the heating times for $n=14$ and 19 arises because of the behavior of the Bessel function for small arguments; $J_{v}(Z) \cong(e Z / 2 v)^{\nu}, e=2.7$.

Table 5 shows an interesting feature of the heating time for nonstochastic situations; the differential heating time reduces drastically with increasing perpendicular energy. Thus, if the ions have an initial perpendicular energy a few times $W_{\perp B}$, the heating time is reduced drastically. For example, when $n=14$, the heating time reduces to about $1 \mathrm{~s}$ when the initial energy of the $\mathrm{O}^{+}$ions is about $3 W_{\perp B}$. Therefore, in order for nonstochastic acceleration to be effective the $\mathrm{O}^{+}$ions must undergo a preacceleration.

Pre-acceleration of ions below $5000 \mathrm{~km}$ is possible; $\mathrm{O}^{+}$ ions accelerated to energies of the order of $100 \mathrm{eV}$ have been reported by Whalen et al. [1978] in the topside ionosphere and by Klumpar [1979] and Ungstrup et al. [1979] at about $1400 \mathrm{~km}$. Chang and Coppi [1981] have shown that the $\mathrm{O}^{+}$ions can be appreciably energized by LHR waves excited by electron beams below $5000 \mathrm{~km}$ in the auroral region.

\subsection{Pitch Angles}

The pitch angles of the ions resulting from the energization process are also given in Tables 2-4, assuming that the parallel energy of $\mathrm{O}^{+}$ions is about $1 \mathrm{keV}$. Note that, depending on the value of $E_{\circ}$ and on the energy (temperature) of the background $\mathrm{H}^{+}$plasma, a range of pitch angles is possible. The conics observed from the S3-3 satellite at altitudes above $5000 \mathrm{~km}$ [e.g., see Sharp et al., 1977] have their angles of maximum flux falling in the range shown in Tables 2-4.

\section{EnERgization of $\mathrm{He}^{+}$}

The important parameters controlling the energization of $\mathrm{He}^{+}$ions are

$$
\begin{array}{ll}
M\left(\mathrm{He}^{+}\right) / M_{\mathrm{H}}=4 & \alpha=K_{\perp}{ }^{2} \Phi_{\circ} M\left(\mathrm{He}^{+}\right) / M_{\mathrm{H}} \\
\nu=\omega / \Omega\left(\mathrm{He}^{+}\right) \cong 5 & \delta=v \alpha\left|J_{5}\left(\zeta_{p, \nu}\right)\right| / \zeta_{p, \nu} \gtrsim 1 / 4
\end{array}
$$

The minimum value of the normalized wave potential amplitude that satisfies the stochasticity condition is found to be

$$
\Phi_{\min }=\phi_{o} / W_{\perp B} \cong 0.22
$$

Comparing (29) and (23), we note that in order to make the motion of $\mathrm{He}^{+}$ions stochastic, a wave amplitude nearly 3 times larger than that for $\mathrm{O}^{+}$ions is required.

\subsection{Energetics}

Table 6 shows the energization of $\mathrm{He}^{+}$ions by using the wave parameters for $E_{\circ}=100 \mathrm{mV} / \mathrm{m}$ shown in Table 1 . Note that only when $W_{\perp B} \lesssim W_{\mathrm{BM}} \cong 20 \mathrm{eV}$ is the stochasticity condition (29) satisfied. Correspondingly, if the value of $E_{0}$ is reduced to $50 \mathrm{mV} / \mathrm{m}, W_{\mathrm{BM}} \cong 10 \mathrm{eV}$. Thus, stochastic acceleration of $\mathrm{He}^{+}$ions is possible either for a cold background $\mathrm{H}^{+}$plasma or for large electric field wave amplitude ( $>50 \mathrm{mV} / \mathrm{m}$ ).

Table 6 shows that for stochastic conditions the $\mathrm{He}^{+}$ perpendicular energy gain extends to only a few hundred $\mathrm{eV}$, while $\mathrm{O}^{+}$ions can be energized to several $\mathrm{keV}$ for the same value of $E_{o}$. Such differences in the energies of $\mathrm{O}^{+}$ and $\mathrm{He}^{+}$ions were recently reported by Lundin et al. [1982].

When the wave amplitude $\Phi_{0}<\Phi_{\text {min }}$, it is still possible to energize the ions corresponding to the first zero of a Bessel function $J_{n}(\xi)$, where the integer $n$ depends on the cyclotron resonances, as discussed previously for $\mathrm{O}^{+}$. The cyclotron resonant parallel energy of the ion is given by (25). Assuming that $\mathrm{He}^{+}$ions have a parallel energy nearly the same as $\mathrm{H}^{+}$ions, it can be shown from (25) that the smallest value of $n$ is an integer close to $2 \hat{\omega}$. However, depending on the parallel energy distribution of $\mathrm{He}^{+}$ions, $n$ may range from 2 to 5 . The range of values of $W_{\perp \max }$ for $W_{\perp B} \gtrsim 50 \mathrm{eV}$ in Table 6 corresponds to the energies of ions given by the first zeros of $J_{2}(\xi)$ and $J_{5}(\xi)$. We note that even for nonstochasticity, it is possible for $\mathrm{He}^{+}$ions to gain an appreciable perpendicular energy through the interaction with a coherent EHC wave, if the background $\mathrm{H}^{+}$ions are hot or if $E_{o}$ is relatively small.

The pitch angles of the ions resulting from the acceleration process are given in Table 6, assuming that their 


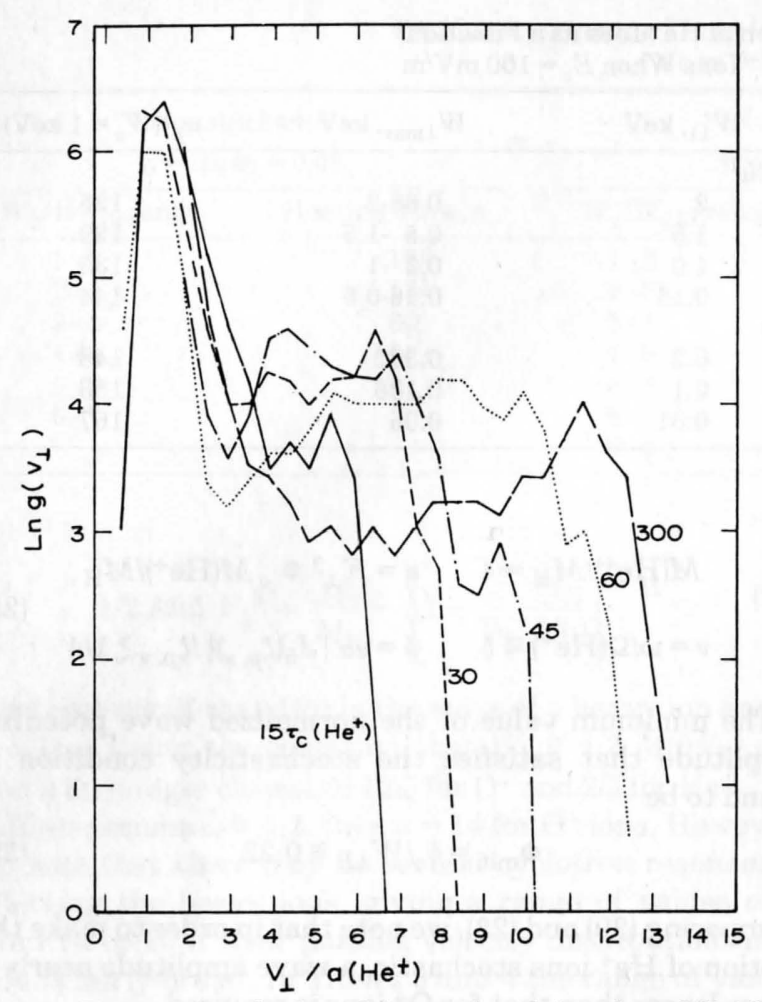

Fig. 4. Temporal evolution of the stochastic heating of $\mathrm{He}^{+}$ions is shown. The initial temperature of the ions is $T\left(\mathrm{He}^{+}\right)=0.1 T_{\mathrm{H}}$. $g\left(V_{\perp}\right)$ is the perpendicular velocity distribution function.

parallel energy is $1 \mathrm{keV}$. Note that the pitch angles vary from $167^{\circ}$ to $125^{\circ}$, depending on the normalized wave amplitude $\Phi_{0}$.

\subsection{Temporal Behavior of the Heating of $\mathrm{He}^{+}$Ions}

Our numerical calculations [Singh et al., 1981] have shown that when the threshold condition (26) is satisfied, leading to stochasticity, $\mathrm{He}^{+}$ions are accelerated very rapidly. Figure 4 shows an example of the heating of $\mathrm{He}^{+}$ ions for $\Phi_{o}=0.4$ and $k_{\perp} / k_{\|}=6$. The temporal evolution of the tail of the distribution function shows that most of the heating has occurred within a time interval of about $60 \tau_{c}\left(\mathrm{He}^{+}\right)$, which is about $1.5 \mathrm{~s}$ at an altitude of $5000 \mathrm{~km}$ on auroral field lines.

When the stochasticity condition (29) is not satisfied, the heating time depends on the ion-cyclotron resonance, as given by (27). However, we note that for $\mathrm{He}^{+}$ions the acceleration occurs very rapidly, unlike that for the heavy $\mathrm{O}^{+}$ions. The heating time at an altitude of about $5000 \mathrm{~km}$ turns out to be less than a second for $\mathrm{He}^{+}$ions.

\section{ENERGIZATION OF $\mathrm{H}^{+}$IONS}

We find that the stochasticity condition for $\mathrm{H}^{+}$ions is not satisfied even in the first cell of $J_{1}(\xi)$ unless the wave amplitude is excessively large $\left(\Phi_{0} \gtrsim 1\right)$. Thus, the energization of $\mathrm{H}^{+}$ions by an EHC wave is limited by the first zero of $J_{1}(\xi)$; the maximum energy gain by a cyclotron resonant ion is

$$
W_{\perp}\left(\mathrm{H}^{+}\right) \cong 7 W_{\perp B}
$$

If we recall from our previous discussion that $\mathrm{EHC}$ waves are excited by the $\mathrm{H}^{+}$ion beam through a Landautype resonance $\omega \cong k_{\|} V_{\|}$, it can be easily seen that the ion beam will not undergo a cyclotron resonance with the wave. So, it is not likely that $\mathrm{H}^{+}$ions will gain perpendicular energy from the coherent wave.

To summarize our discussion so far, we emphasize that the perpendicular energy gain of ions through the interaction with a coherent EHC wave is highly mass dependent; the heavier the ion, the larger is the energy gain.

\section{ACCEleration OF IONS By Higher HaRMonics OF EHC WAVES}

In a recent paper, Temerin et al. [1982] reported observations of 2nd and 3rd harmonics of $\Omega_{\mathrm{H}}$ with wave amplitudes comparable to that of the fundamental. Under such a situation, harmonics can play an even greater role in the acceleration of the heavy ions than the fundamental itself. Such harmonic waves are characterized by wave frequencies and wave numbers given by

$$
\omega_{m} \cong m \Omega_{\mathrm{H}} \text { and } k_{\perp} r_{\mathrm{H}} \cong m \quad m=2,3,4--
$$

where $m$ is the harmonic number. When these wave parameters are used in the formulas describing the acceleration of $\mathrm{O}^{+}$ions, we obtain

$$
\begin{aligned}
& \alpha \cong m^{2} \Phi_{0} M\left(\mathrm{O}^{+}\right) / M_{\mathrm{H}}, \nu \cong m M\left(\mathrm{O}^{+}\right) / M_{\mathrm{H}} \\
& \delta=m^{3}\left[\frac{M\left(\mathrm{O}^{+}\right)}{M_{\mathrm{H}}}\right]^{2} \Phi_{o}\left|J_{v}\left(\zeta_{p, v}\right)\right| / \zeta_{p, v} \geq 1 / 4
\end{aligned}
$$

If the wave amplitude is large enough so that $J_{\nu}(\zeta)$ can be replaced by its asymptotic expression, the maximum energy gain is given by

$$
E_{\max } \cong 3.2(2 / \pi)^{2 / 3} m^{2} \phi_{0}^{4 / 3}\left(M\left(\mathrm{O}^{+}\right) / M_{\mathrm{H}}\right)^{5 / 3} W_{\perp B}
$$

Comparing (18) with (33) we note that when $k_{\perp} r_{\mathrm{H}} \cong 1$ for the fundamental, the maximum energy gain scales as $m^{2}$, provided that the wave amplitude $\hat{\phi}_{o}$ is nearly the same for the harmonics. Temerin et al. [1981] reported that, at times, only the second harmonic was seen with an electric field wave amplitude of about $20 \mathrm{mV} / \mathrm{m}$. Such a wave can energize $\mathrm{O}^{+}$ions more than the energies $W_{\perp \max }$ given in Table 4 for an EHC wave with the fundamental harmonic of the hydrogen cyclotron frequency.

\section{Discussion AND CONCLUSION}

We have shown that preferential acceleration of heavy ions occur when such ions interact with an EHC wave. Under both stochastic and nonstochastic situations, $\mathrm{O}^{+}$ and $\mathrm{He}^{+}$ions gain appreciable transverse energy from the wave. The calculation of the heating time showed that $\mathrm{O}^{+}$ and $\mathrm{He}^{+}$ions gain most of their energy in about $2 \mathrm{~s}$ for stochastic acceleration. However, when the stochasticity condition is not met, $\mathrm{He}^{+}$ions still undergo a rapid heating, while $\mathrm{O}^{+}$ions take several hours to heat if their initial energy $W_{\perp} \cong W_{\perp B}$. But it is interesting to note that for $\mathrm{O}^{+}$ions the stochasticity condition is met at much lower wave amplitudes $\left(\phi_{0} \cong 0.07\right)$ than that for the $\mathrm{He}^{+}$ 
ions. Consequently, it can be argued that $\mathrm{O}^{+}$ions are preferentially accelerated compared to $\mathrm{He}^{+}$ions.

We now compare the extent of transverse energization of $\mathrm{O}^{+}$and $\mathrm{He}^{+}$obtained in this paper with the observations of Collin et al. [1981] and Lundin et al. [1982]. Figure 5 shows the correlation between the average energies of $\mathrm{O}^{+}$and $\mathrm{H}^{+}$ions in some UFI events, as given in Figure 4 of Collin et al. [1981]. Note the scatter of the points above the line $W\left(O^{+}\right)=W\left(\mathrm{H}^{+}\right)$. The additional energy of $\mathrm{O}^{+}$ions above this line is believed to be the perpendicular energy gained by $\mathrm{O}^{+}$from some preferential transverse acceleration mechanism. We suggest that this mechanism is the interaction of $\mathrm{O}^{+}$ions by an EHC wave excited by the $\mathrm{H}^{+}$ion beam in the same UFI event.

Assuming that the UFI events occurred under conditions when the background $\mathrm{H}^{+}$temperature varied from 1 to $200 \mathrm{eV}$, we find that the scatter of points in Figure 5 can be accounted for when the electric field wave amplitude is assumed to be $E_{o} \cong 50 \mathrm{mV} / \mathrm{m}$. The dotted line in Figure 5 is given by $W\left(\mathrm{O}^{+}\right)=W\left(\mathrm{H}^{+}\right)+W_{\perp \max }$, where $W_{\perp \text { max }}$ is evaluated for $W_{\perp B}=200 \mathrm{eV}$ and $E_{o}=50 \mathrm{mV} / \mathrm{m}$. For other values of $W_{\perp B}<200 \mathrm{eV}$ and $E_{o}<50 \mathrm{mV} / \mathrm{m}$, the energy gain $W_{\perp \max }$ fall below the dotted line. Thus, it appears that the scatter of points representing the additional energy gain by $\mathrm{O}^{+}$ions can be simulated by varying both $W_{\perp B}$ and $E_{\mathrm{o}}$ in a random fashion. The upper bounds on $E_{0}$ and $W_{\perp B}$ seem reasonable from the observational point of view.

We have made a similar comparison between the data of Lundin et al. [1982] and our theory for $\mathrm{He}^{+}$ions. The results are shown in Figure 6, which is the same as Figure 7 of these authors. We note that the theory using values of $W_{\perp B} \lesssim 200 \mathrm{eV}$ and $E_{\circ}<100 \mathrm{mV} / \mathrm{m}$ describes fairly well the additional energization of $\mathrm{He}^{+}$ions above the energy of the $\mathrm{H}^{+}$ions.

The most significant contribution of this paper is to demonstrate clearly that with the observed electric field wave amplitudes $E_{\text {o }}$ of a few tens of $\mathrm{mV} / \mathrm{m}$, the preferential acceleration of heavy ions by EHC waves is possible. For a given value of $E_{0}$, the extent of energization critically depends on the average perpendicular energy of the background $\mathrm{H}^{+}$ions supporting the wave. This dependence stems from the fact that the wavelength of

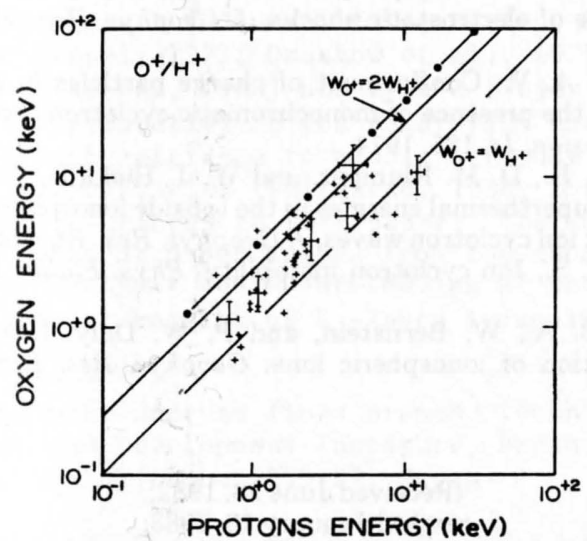

Fig. 5. Scatter plot of average $\mathrm{O}^{+}$and $\mathrm{H}^{+}$energies in upflowing ion beams [Collin et al., 1981]. The broken line with dots is obtained by plotting the value of $W_{\perp \text { max }}$ in Table 3 for $W_{\perp B}=200$ eV above the line $W\left(\mathrm{O}^{+}\right)=W\left(\mathrm{H}^{+}\right)$

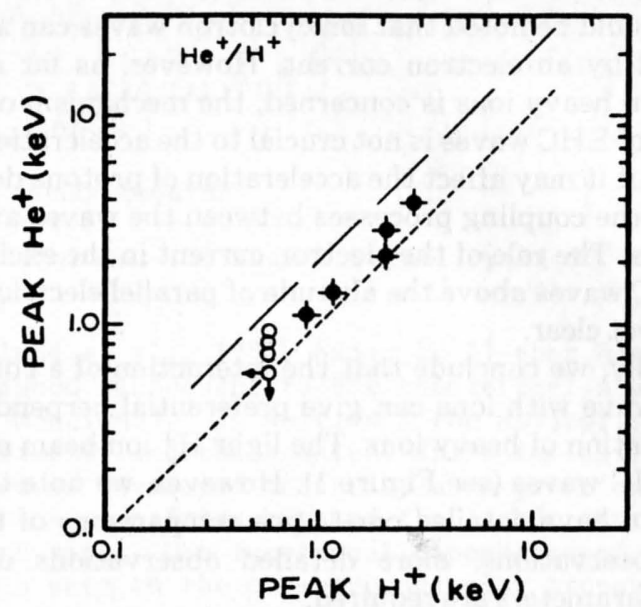

Fig. 6. Peak energy for $\mathrm{He}^{+}$plotted versus the $\mathrm{H}^{+}$peak energy [Lundin et al., 1982]. The dashed-broken line is obtained by plotting $W_{\perp \max }$ in Table 6 for $W_{\perp B}=200 \mathrm{eV}$ above the line $W\left(\mathrm{He}^{+}\right)=$ $W\left(\mathrm{H}^{+}\right)$.

the waves is determined by the average Larmor radius of these ions.

Calculations of the heating time showed that typically $\mathrm{O}^{+}$and $\mathrm{He}^{+}$ions are energized in about $2 \mathrm{~s}$ at an altitude of about $5000 \mathrm{~km}$ when the wave amplitudes are sufficiently large to create stochasticity in the motion of the ions. Otherwise, the heating is slow for $\mathrm{O}^{+}$ions. The threshold wave amplitude beyond which fast heating is possible for $\mathrm{O}^{+}$is given by (23). For $\mathrm{He}^{+}$ions the heating is fast even in the nonstochastic situation.

Previously, Lysak et al. [1980] and Dusenbery and Lyons [1981] investigated the acceleration of ions by ioncyclotron waves. In these studies it was shown that a particular ion species can be accelerated by ion-cyclotron waves supported by the same species. On the other hand, in this paper preferential accelerations of relatively heavy ions by EHC waves is shown. Such waves have been observed from the S3-3 satellite [Kintner et al., 1978, 1979; Temerin et al., 1981].

Another important contrasting feature of our paper with those of Lysak et al. [1980] and Dusenbery and Lyons [1981] is that the ion cyclotron resonances are not crucial for the acceleration of ions when the wave amplitudes are sufficiently large to cause stochasticity. This point has been discussed in detail previously by Singh et al. [1982b]. However, we note that when the wave amplitudes are small (see (24) and (28)), the acceleration occurs only when the ions have a cyclotron resonance with the wave. If the wave is excited by an $\mathrm{H}^{+}$ion beam through the resonance condition $\omega \cong k_{\|} V_{\|}$, which appears to be the case above $5000 \mathrm{~km}$ in the auroral plasma, the $\mathrm{H}^{+}$beam ions cannot undergo a cyclotron resonance with the wave. Thus, these ions cannot gain a perpendicular energy from the EHC wave.

It is worth pointing out that there is an alternative mechanism for exciting $\mathrm{EHC}$ waves by an $\mathrm{H}^{+}$ion beam; in this mechanism the cyclotron modes of the background and the beam ions interact to cause instability [Weibel, 1970] In such a situation, beam ions are cyclotron resonant, and then part of the beam energy $W_{o}$ can be converted into its perpendicular energy as determined by (30), if the excited wave is coherent. 
It should be noted that ion cyclotron waves can also be excited by an electron current. However, as far as our work on heavy ions is concerned, the mechanism of excitation of EHC waves is not crucial to the acceleration process, but it may affect the acceleration of protons depending on the coupling processes between the waves and the protons. The role of the electron current in the excitation of $\mathrm{EHC}$ waves above the altitude of parallel electric fields is not yet clear.

Finally, we conclude that the interaction of a coherent $\mathrm{EHC}$ wave with ions can give preferential perpendicular acceleration of heavy ions. The light $\mathrm{H}^{+}$ion beam excites the EHC waves (see Figure 1). However, we note that in order to have detailed one-to-one comparisons of theory and observations, more detailed observations on the wave parameters are required.

Acknowledgements. This research was supported by NASA grant NAGW-77 and NSF grant ATM-8015497 to Utah State University.

The Editor thanks the two referees for their assistance in evaluating this paper.

\section{REFERENCES}

Banks, P. M., and T. E. Holzer, High-latitude plasma transport: The polar wind, J. Geophys. Res., 74, 6317, 1969.

Baugher, C. R., C. R. Chappell, J. L. Horwitz, E. G. Shelley, and D. T. Young, Initial thermal plasma observations from ISEE 1, Geophys. Res. Lett., 7, 657, 1980.

Chang, T., and B. Coppi, Lower hybrid acceleration and ion evolution in the suprauroral region, Geophys. Res. Lett., 8, $1253,1981$.

Collin, H. L., R. D. Sharp, E. G. Shelley, and R. G. Johnson, Some general characteristics of upflowing ion beams over the auroral zone and their relationship to auroral electrons, $J$. Geophys. Res., 86, 6820, 1981.

Comfort, R. H., and J. L. Horwitz, Low-energy ion pitch angle distributions on the dayside at synchronous orbit, J. Geophys. Res., 86, 1621, 1981.

Dusenbery, P. B., and L. R. Lyons, Generation of ion-conic distribution by upgoing ionospheric electrons, $J$. Geophys. Res., 86, 7627, 1981.

Frank L. A., K. L. Ackerson, and D. M. Yeager, Observations of atomic oxygen $\left(\mathrm{O}^{+}\right)$in the earth's magnetotail, J. Geophys. Res., 82, 129, 1977.

Fredericks, R. W., F. L. Scarf, and W. Bernstein, Numerical estimates of superthermal electron production by ion acoustic waves in the transition region, J. Geophys. Res., 70, 21, 1965.

Fukuyama, A., H. Momota, R. Itatani, and T. Takizuka, Stochastic acceleration by an electrostatic wave near ion cyclotron harmonics, Phys. Rev. Lett., 38, 701, 1977.

Gorney, D. J., A. Clarke, D. Croley, J. Fennell, J. Luhmann, and P. Mizera, The distribution of ion beams and conics below $8000 \mathrm{~km}, J$. Geophys. Res., 86, 83, 1981.

Hardy, D. A., J. W. Freeman, and H. K. Hills, Double-peaked ion spectra in the wake plasma: Evidence of massive ions?, $J$. Geophys. Res., 82, 5529, 1977.

Horwitz, J. L., and C. R. Chappell, Observations of warm plasma in the dayside plasma trough at geosynchronous orbit, J. Geophys. Res., 84, 7075, 1979.

Horwitz, J. L., C. R. Baugher, C. R. Chappell, E. G. Shelley, and D. T. Young, Pancake pitch angle distributions in warm ions observed with ISEE 1,J. Geophys. Res., 86, 3311, 1981.

Karney, C. F. F., Stochastic ion heating by a lower hybrid wave, Phys. Fluids, 21, 1584, 1978.

Karney, C. F. F., Stochastic ion heating by a lower hybrid wave: II, Phys. Fluids, 22, 2188, 1979.
Karney, C. F. F., and A. Bers, Stochastic ion heating by a perpendicularly propagating electrostatic wave, Phys. Rev. Lett., 39, 550, 1977.

Kintner, P. M., On the distinction between electrostatic ion cyclotron waves and ion cyclotron harmonic waves, Geophys. Res. Lett., 7, 585-588, 1980.

Kintner, P. M., M. C. Kelley, and F. S. Mozer, Electrostatic hydrogen cyclotron waves near one earth radius altitude in the polar magnetosphere, Geophys Res. Lett., 5, 139, 1978.

Kintner, P. M., M. C. Kelley, R. D. Sharp, A. G. Ghielmetti, M. Temerin, C. Cattell, P. F. Mizera, and J. F. Fennell, Simultaneous observations of energetic $(\mathrm{keV})$ upstreaming ions and electrostatic hydrogen cyclotron waves, J. Geophys. Res., 84, 7201, 1979.

Klumpar, D. M., Transversely accelerated ions: An ionospheric source of hot magnetospheric ions, J. Geophys. Res., 84, 4229, 1979.

Lundin, R., B. Hultquist, E. Dubinin, and B. Pissarenko, Observations of outflowing ion beams on auroral field lines at altitudes of many earth radii, Planet. Space Sci., 30, 715, 1982.

Lysak, R. L., M. K. Hudson, and M. Temerin, Ion heating by strong electrostatic ion cyclotron turbulence, J. Geophys. Res., 85, 678, 1980.

Papadopoulos, K., J. D. Gaffey, Jr., and P. J. Palmadesso, Stochastic acceleration of large $M / Q$ ions by hydrogen cyclotron waves in the magnetosphere, Geophys, Res. Lett., 7, $1014,1980$.

Perkins, F. W., Ion-streaming instabilities: Electromagnetic and electrostatic, Phys. Fluids, 19, 1012-1020, 1976.

Sharp, R. D., R. G. Johnson, and E. G. Shelley, Observation of an ionospheric acceleration mechanism producing energetic $(\mathrm{keV})$ ions primarily normal to the geomagnetic field direction, J. Geophys. Res., 82, 3324, 1977.

Shelley, E. G., Heavy ions in the magnetosphere, Space Sci. Rev., 23, 465, 1979.

Singh, N., R. W. Schunk, and J. J. Sojka, Energization of ionospheric ions by electrostatic hydrogen cyclotron waves, Geophys. Res. Lett., 8, 1249, 1981.

Singh, N., W. J. Raitt, and F. Yasuhara, Low-energy ion distribution functions on a magnetically quiet day at geostationary altitude $(L=7), J$. Geophys. Res., 87, 681-694, $1982 a$.

Singh, N., R. W. Schunk, and J. J. Sojka, Cyclotron resonance effects on stochastic acceleration of light ionospheric ions, Geophys. Res. Lett., 9, 1053-1056, $1982 b$.

Smith, R., and A. N. Kaufman, Stochastic acceleration by a single wave in a magnetic field, Phys. Rev. Lett., 34, 1613, 1975.

Smith. G. R., and A. N. Kaufman, Stochastic acceleration by an obliquely propagating wave-An example of overlapping resonances, Phys. Fluids, 21, 2230, 1978.

Stix, T. H., Energetic electrons from a beam-plasma overstability, Rep. MATT-239, Plasma Phys. Lab., Princeton Univ., Princeton, N.J., February 1965.

Temerin, M., C. Cattell, R. Lysak, M. Hudson, R. B. Torbet, F. S. Mozer, R. D. Sharp, and P. M. Kintner, The small-scale structure of electrostatic shocks, J. Geophys. Res., 86, 11278, 1981.

Timofeev, A. V., Confinement of charge particles in adiabatic traps in the presence of monochromatic cyclotron oscillations, Nucl. Fusion, 14, 165, 1974.

Ungstrup, E., D. M. Klumpar, and W. J. Heikkila, Heating of ions to superthermal energies in the topside ionosphere by electrostatic ion cyclotron waves, J. Geophys. Res., 84, 4289, 1979.

Weibel, E. S., Ion cyclotron instability, Phys. Fluids, 13, 3003, 1970.

Whalen B. A., W. Bernstein, and P. W. Daly, Low-altitude acceleration of ionospheric ions, Geophys. Res. Lett., 5, 55, 1978.

(Received June 28, 1982;

revised January 13, 1983;

accepted January 14, 1983.) 